\title{
Numerical Simulation on Mixed Convection Flow within Triangular Enclosures Having Heatline Concept
}

\author{
Muhammad Sajjad Hossain ${ }^{1}$, M. Saiful Islam Mallik ${ }^{1}$ and M. A. Rob ${ }^{2}$ \\ ${ }^{1}$ Assistant Professor of Mathematics, Department of Arts and Sciences, Ahsanullah University of Science and \\ Technology (AUST), Dhaka-1208, Bangladesh \\ ${ }^{2}$ Lecturer in Mathematics, Department of Electrical and Electronic Engineering, Eastern University \\ Dhaka-1205, Bangladesh
}

\begin{abstract}
This paper is conducted to investigate mixed convection flow within triangular enclosures having heatline concept. Here the left vertical wall which is moving from the bottom corner of the enclosure is also kept at a uniform constant cold temperature and the bottom wall is heat generating, while the other inclined wall is adiabatic. A finite element analysis is performed for solving the governing equations which are discretized by Galerkin weighted residual method of finite element formulation. The present numerical procedure adopted in this investigation yields consistent performance over a wide range of parameters Prandtl number, $\operatorname{Pr}(\operatorname{Pr}=0.71$ - 6) varying Rayleigh number (Ra) from $10^{3}$ to $10^{4}$ while Reynolds number, Re $(R e=50)$ is fixed. The Result also indicates streamlines, isotherms, average Nusselt number along the hot wall, average temperature of the fluid in the enclosure. The computational results also indicate that the average Nusselt number at the hot wall of the Enclosure is depending on the dimensionless parameters.

Keywords: Mixed convection, Finite element method, Triangular enclosure, Heatline.
\end{abstract}

\section{Introduction}

Modification and control of flow and heat transfer in a triangular enclosure is a viable approach in various engineering applications. Analysis of mixed convection usually induced in enclosed Enclosures or channels containing heating elements on one of its wall or on both walls are important from both theoretical and practical points of view. Most of the common enclosures used in industries are cylinder, rectangular, trapezoidal and triangular. However triangular enclosures have received a considerable attention in various fields. A brief review of the relevant literature is presented in the following section.

In mixed convection, structures of laminar wakes and heat transfer in a horizontal channel with a builtin square cylinder were studied numerically by Biswas et al. [1]. In their study, they found that the channel walls and the surface of the bluff body have higher temperatures than the incoming flow and the mixed convection initiate periodicity and asymmetry in the wake at a lower $R e$ than forced convection alone. Moreover, Raji and Hasnaoui ([2],[3]) obtained numerical results by using a finite difference procedure for opposing flows mixed (forced and natural) convection flow in a rectangular Enclosure heated from the side with a constant heat flux and submitted to a laminar cold jet from the bottom of its heated wall. Also, Omri and Nasrallah [4] performed numerical analysis by a control volume finite element method on mixed convection in a rectangular enclosure with differentially heated vertical sidewalls. Later on, Raji and Hasnaoui [5] investigated the mixed convection in ventilated Enclosures where the horizontal top wall and the vertical left wall were prescribed with equal heat fluxes. At the same time, Angirasa [6] numerically studied and explained the complex interaction between buoyancy and forced flow in a square enclosure with an inlet and a vent situated respectively, at the bottom and top edges of the vertical isothermal surface, where the other three walls are adiabatic. Singh and Sharif [7] extended their works by considering six placement configurations of the inlet and outlet of a differentially heated rectangular enclosure whereas the previous work was limited to only two different configurations of inlet and outlet. Hossain and Gorla [8] investigated the effects of viscous dissipation on unsteady combined convective heat transfer to water near its density maximum in a rectangular Enclosure with isothermal wall. Varol et al. [9] studied free convection in porous media right-angle triangular enclosure. They considered steady-state free convection heat transfer in a right-angle triangular enclosure, whose vertical wall insulated and inclined and bottom walls are differentially heated. In this study they showed the effect of aspect ratios ranging from 0.25 to 1.0 and Rayleigh numbers $50 \leq R a \leq 1000$ was investigated as governing parameters on heat transfer and flow field. A Finite Element Analysis of natural convection flow in an isosceles triangular enclosure due to uniform and non-uniform heating at the side walls was studied by Basak et al. [10]. In this study they used penalty finite element analysis with bi-quadratic elements. Two cases of thermal boundary conditions are considered Case-1: two inclined walls are uniformly heated while the bottom wall is cold isothermal and Case2: two inclined walls are non-uniformly heated while the bottom wall is cold isothermal. The numerical solution of the problem was presented for various Rayleigh numbers $\left(10^{3} \leq R a \leq 10^{6}\right)$ and Prandlt numbers 
$(0.007 \leq P r \leq 1000)$. They found that at small Prandlt numbers, geometry does not have much influence on flow structure while at $\operatorname{Pr}=1000$, geometry has considerable effect on the flow pattern. Recently Rahman et al. [11] studied numerically the opposing mixed convection in a vented enclosure. They found that with the increase of Reynolds and Richardson numbers the convective heat transfer becomes predominant over the conduction heat transfer and the rate of heat transfer from the heated wall is significantly depended on the position of the inlet port.

Our main goal is to find numerical simulation on mixed convection flow within triangular Enclosures having heatline concept. Results are presented in terms of streamlines, isotherms, average nusselt number along the hot wall, average temperature of the fluid in the Enclosure and dimensionless temperature at the cylinder center for Prandtl number $\mathrm{Pr}$, and at the three values of Rayleigh number $R a$, varying from $10^{3}$ to $10^{4} \mathrm{keeping}$ fixed Reynolds number, $R e$.

\section{Mathematical Model}

Figure 1 shows the heat transfer and the fluid flow in a two-dimensional triangular Enclosure with a fluid whose left wall and bottom wall are cold $T_{c}$ and hot $T_{h}$ temperatures respectively while the inclined walls are kept adiabatic. The model describes with Prandtl number $(\operatorname{Pr}=0.71-6)$, Reynolds number $(\operatorname{Re}=50)$ and Newtonian and the fluid flow is considered to be laminar and its properties were constant.

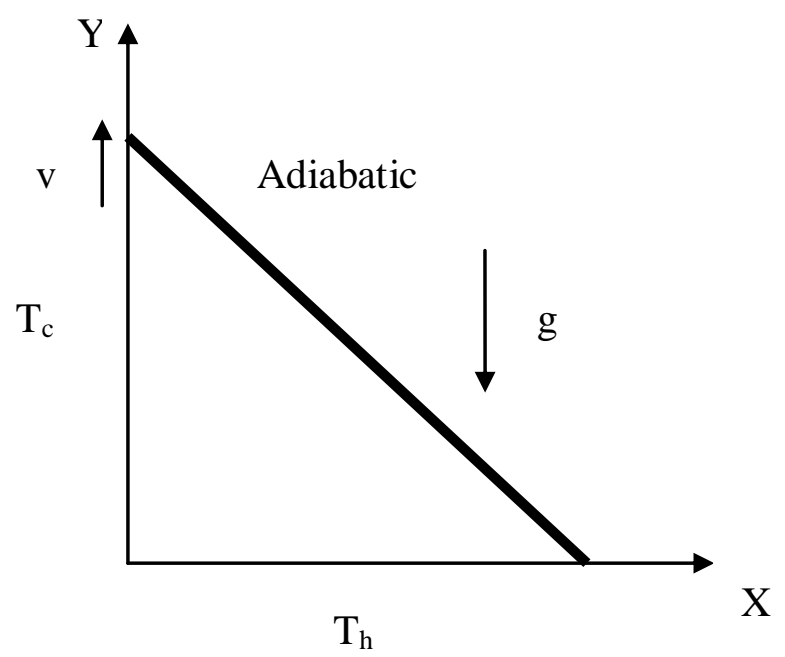

Figure1: Schematic diagram of the physical system

\section{Governing Equations}

Two-dimensional, steady, laminar and incompressible flow is assumed inside the Enclosure and the fluid properties are constant. In this Problem, the buoyancy force is included here as a body force in the $v$ momentum equation. The non-dimensional governing equations describing the flow can be expressed as:

$$
\begin{aligned}
& \frac{\partial U}{\partial X}+\frac{\partial V}{\partial Y}=0 \\
& U \frac{\partial U}{\partial X}+V \frac{\partial U}{\partial Y}=-\frac{\partial P}{\partial X}+\frac{1}{R e}\left(\frac{\partial^{2} U}{\partial X^{2}}+\frac{\partial^{2} U}{\partial Y^{2}}\right) \\
& U \frac{\partial U}{\partial X}+V \frac{\partial U}{\partial Y}=-\frac{\partial P}{\partial Y}+\frac{1}{\operatorname{Re}}\left(\frac{\partial^{2} V}{\partial X^{2}}+\frac{\partial^{2} V}{\partial Y^{2}}\right)+R a \operatorname{Pr} \theta \\
& U \frac{\partial \theta}{\partial X}+V \frac{\partial \theta}{\partial Y}=\frac{1}{\operatorname{Re} \operatorname{Pr}}\left(\frac{\partial^{2} \theta}{\partial X^{2}}+\frac{\partial^{2} \theta}{\partial Y^{2}}\right)
\end{aligned}
$$

The following dimensionless Parameters are used in writing equations (1- 4) as: 


$$
\begin{aligned}
& X=\frac{x}{L}, Y=\frac{y}{L}, U=\frac{u}{v_{0}}, V=\frac{v}{v_{0}}, P=\frac{p}{\rho v_{0}^{2}}, \theta=\frac{\left(T-T_{c}\right)}{\left(T_{h}-T_{c}\right)}, \operatorname{Re}=\frac{u_{i} L}{v}, \operatorname{Pr}=\frac{v}{\alpha}, \\
& G r=\frac{\beta g \Delta T L^{3}}{v^{2}}, \operatorname{Ra}=G r \times \operatorname{Pr}, \Delta T=T_{h}-T_{c}, \alpha=\frac{\kappa}{\rho C_{p}}
\end{aligned}
$$

The appopriate boundary conditions for the above equations (1)-(4), are considered as:

At the left vertical wall: $U=0, V=1, \theta=0$

At the bottom wall: $U=0, V=0, \theta=1$

At the inclined wall: $U=0, V=0, \frac{\partial \theta}{\partial N}=0$

According to Singh and Sharif [7], the average Nusselt number at the heated wall of the Enclosure based on the non-dimensional variables may be expressed as $N u=\int_{0}^{1}\left(\frac{\partial \theta}{\partial Y}\right)_{y=0} d X$ and the bulk average temperature defined as $\theta_{a v}=\int \theta d \bar{V} / \bar{V}$, where $\bar{V}$ is the Enclosure volume.

\section{Numerical Analysis}

The momentum and energy balance equations are the combination of mixed elliptic-parabolic system of partial differential equations that have been solved by using the Galerkin weighted residual finite element technique of Rahman et al. [12]. The basic unknowns for the above differential equations are the velocity components $U, V$, the temperature $\theta$ and the pressure $P$. The velocity components, the temperature distributions and linear interpolation for the pressure distribution according to their highest derivative orders in the differential eqs. (1) - (4) are as follows:

$U(X, Y)=N_{\alpha} U_{\alpha}, \quad V(X, Y)=N_{\alpha} V_{\alpha}, \theta(X, Y)=N_{\alpha} \theta_{\alpha}, P(X, Y)=H_{\lambda} P_{\lambda}$

where $\alpha=1,2, \ldots \ldots, 6 ; \lambda=1,2,3 ; \mathrm{N}_{\alpha}$ are the element interpolation functions for the velocity components and the temperature, and $\mathrm{H}_{\lambda}$ are the element interpolation functions for the pressure.

To derive the finite element equations, the method of weighted residuals finite-element formulation is applied to the equations (1) - (4) as

$$
\begin{aligned}
& \int_{A} N_{\alpha}\left(\frac{\partial U}{\partial X}+\frac{\partial V}{\partial Y}\right) d A=0 \\
& \int_{A} N_{\alpha}\left(U \frac{\partial U}{\partial X}+V \frac{\partial U}{\partial Y}\right) d A=-\int_{A} H_{\lambda}\left(\frac{\partial P}{\partial X}\right) d A+\frac{1}{R e} \int_{A} N_{\alpha}\left(\frac{\partial^{2} U}{\partial X^{2}}+\frac{\partial^{2} U}{\partial Y^{2}}\right) d A \\
& \int_{A} N_{\alpha}\left(U \frac{\partial V}{\partial X}+V \frac{\partial V}{\partial Y}\right) d A=-\int_{A} H_{\lambda}\left(\frac{\partial P}{\partial Y}\right) d A+ \\
& \frac{1}{\operatorname{Re}} \int_{A} N_{\alpha}\left(\frac{\partial^{2} V}{\partial X^{2}}+\frac{\partial^{2} V}{\partial Y^{2}}\right) d A+R a \operatorname{Pr} \int_{A} N_{\alpha} \theta d A \\
& \int_{A} N_{\alpha}\left(U \frac{\partial \theta}{\partial X}+V \frac{\partial \theta}{\partial Y}\right) d A=\frac{1}{\operatorname{Re} P r} \int_{A} N_{\alpha}\left(\frac{\partial^{2} \theta}{\partial X^{2}}+\frac{\partial^{2} \theta}{\partial Y^{2}}\right) d A
\end{aligned}
$$

So, the coefficients in element matrices are in the form of the integrals over the element area as,

$$
\begin{array}{lll}
K_{\alpha \beta^{x}}=\int_{A} N_{\alpha} N_{\beta, x} d A, & K_{\alpha \beta}^{y}=\int_{A} N_{\alpha} N_{\beta, y} d A & K_{\alpha \beta \gamma} x=\int_{A} N_{\alpha} N_{\beta} N_{\gamma, x} d A \\
K_{\alpha \beta \gamma} y=\int_{A} N_{\alpha} N_{\beta} N_{\gamma, y} d A & K_{\alpha \beta}=\int_{A} N_{\alpha} N_{\beta} d A, & S_{\alpha \beta} x x=\int_{A} N_{\alpha, x} N_{\beta, x} d A
\end{array}
$$




$$
\begin{aligned}
& S_{\alpha \beta}^{y y}=\int_{A} N_{\alpha, y} N_{\beta, y} d A \\
& M_{\alpha \mu^{x}}=\int_{A} H_{\alpha} H_{\mu, x} d A \quad M_{\alpha \mu}^{y}=\int_{A} H_{\alpha} H_{\mu, y} d A \\
& Q_{\alpha} u=\int_{S_{0}} N_{\alpha} S_{x} d S_{0} \\
& Q_{\alpha} v=\int_{S_{0}} N_{\alpha} S_{y} d S_{0} \text {, } \\
& Q_{\alpha} \theta=\int_{S_{w}} N_{\alpha} q_{1_{w}} d S_{w} \\
& Q_{\alpha} \theta_{s}=\int_{S_{w}} N_{\alpha} q_{2 w} d S_{w}
\end{aligned}
$$

Using reduced integration technique of Reddy [13] and Newton-Raphson iteration technique by first writing the unbalanced values from the set of the finite element equations, the nonlinear algebraic Eqs. (5) - (8) so obtained are modified by imposition of boundary conditions. These modified nonlinear equations are transferred into linear algebraic equations by using Newton's method. Finally, these linear equations are solved by using Triangular Factorization method.

\section{Grid Refinement Test}

In order to obtain grid independent solution, a grid refinement study is performed for a triangular Enclosure with $\operatorname{Pr}=0.71, R e=50$ and $R a=10^{3}$. Table 1 shows the convergence of the average Nusselt number, $N u_{a v}$ at the heated surface with grid refinement. Five different non-uniform grids are considered for the grid independence test. These are 9138, 22020, 49260, 62280, 83802 nodes and 1523, 3670, 8210, 10380, 13967 elements. From these values 83802 nodes and 13967 elements are chosen for better accuracy to optimize the simulation.

\begin{tabular}{|c|c|c|c|c|c|}
\hline $\begin{array}{c}\text { Nodes } \\
\text { (elements) }\end{array}$ & 9138 & 22020 & 49260 & 62280 & 83802 \\
1523 & 3670 & 8210 & 10380 & 13967 \\
\hline $\mathrm{Nu}$ & 2.4095029 & 2.6431469 & 2.5207523 & 2.6746807 & 2.7484171 \\
\hline Time (s) & 8.297 & 19.609 & 47.703 & 67.297 & 94.828 \\
\hline
\end{tabular}

Table 1: Grid Sensitivity Check at $\operatorname{Pr}=0.71, R e=50$ and $R a=10^{4}$.

\section{Code Validation}

The present numerical code is validated against the numerical results of Basak et al. (2007), for pure natural convection with $P r=0.71$ in a triangular Enclosure. The computed average Nusselt numbers for the constant surface temperature and different Rayleigh Numbers are tabulated in Table 2. The agreement between the present computation and those of Basak et al.(2007), are seen to be very well. These validations make a good confidence in the present numerical code.

\begin{tabular}{|c|c|c|}
\hline \multirow{2}{*}{$R a$} & \multicolumn{2}{|c|}{$N u_{\mathrm{av}}$} \\
\cline { 2 - 3 } & Present work & Basak et al. (2007) \\
\hline $10^{3}$ & 5.49 & 5.40 \\
\hline $10^{4}$ & 5.77 & 5.56 \\
\hline $10^{5}$ & 7.08 & 7.54 \\
\hline
\end{tabular}

Table 2: Comparison between Basak et.al.(2007) and present work

\section{Results and Discussion}

A numerical study has been performed through finite element method to conclude the properties of the presence of dimensionless parameters in triangular Enclosure with a heat conducting horizontal bottom wall and the other two remaining walls were cold Temperature $T_{c}$ (right wall) and adiabatic (inclined wall). Here twodimensional forms of Navier-Stokes equations along with the energy equations are used. The dimensionless are Reynolds number $R e$, Rayleigh number $R a$, Prandtl number $P r$. The numerical results are presented to explain the effect of several parameters at a small fraction of the possible situations by simplifying the configuration. Results are obtained for a wide range of Rayleigh number from $10^{3}$ to $10^{4}$ at $\operatorname{Pr}=0.71-6$ and $R e=50$, with constant physical properties. To obtain stream functions and temperature profiles, the parametric studies for a wide range of governing parameters show consistent performance of the present numerical approach. It is indicating that that the heat transfer coefficient is strongly affected by Rayleigh number. A representative set of numerical results is shown graphically in figs.2 - 5, to illustrate the influence of physical parameters viz., Reynolds number $R e$, Rayleigh number $R a$ and Prandtl number $P r$. 
Fig. $2-5$ display the effects of $\operatorname{Ra}\left(R a=10^{3}-10^{4}\right)$ and $\operatorname{Pr}(\operatorname{Pr}=0.71-6.0)$ when the bottom wall is heated while the left moving wall is maintained at cold temperature. The fluid near to the hot (bottom) wall has lower density, so it moves upward while the relatively heavy fluid near to cold (left) wall moves downward and this fluid is heated up. Thus the fluid motion completes the circulation. As mentioned before by the definition of stream function, the streamlines with ' $+\psi$ ' corresponds to anti-clockwise circulation, and those with ' $-\psi$ ' correspond to clockwise circulation. Note that, for steady flow, stream lines are equivalent to the paths followed by the individual particles in the fluid. Results indicate that at low Rayleigh number $\left(R a=10^{3}\right)$, the isotherm lines are smooth and monotonic as shown for $\operatorname{Pr}=0.71 \mathrm{in} \mathrm{Fig.} \mathrm{2.} \mathrm{The} \mathrm{magnitudes} \mathrm{of} \mathrm{the} \mathrm{stream} \mathrm{function} \mathrm{contours}$ are very low. This illustrates that at low $R a$ the flow is mostly due to conduction.

At $R a=5 \times 10^{3}$ and $\operatorname{Pr}=0.71$, the circulations near the central regime are stronger and consequently, the thermal boundary layer start getting shifted towards the left wall (Fig. 3- 5).The presence of significant convection is also exhibited in the temperature profile which get pushed towards the upper portion of left wall. As Rayleigh number increases to $10^{4}$, the buoyancy driven circulation inside the Enclosure is also increased as seen from greater magnitudes of the stream function (Fig. 2 - 5). Note that, Fig. 5 for $\operatorname{Pr}=6.0$ shows the circulations are bigger near the center due to convection and least at the vicinity wall due to no-slip boundary conditions. The greater circulation in central regime follows a progressive packaging around the center of rotation, and a more and more pronounced compression of the isotherms towards the bottom and left wall of triangular Enclosure occurs. Due to high circulations, the temperature contours with 300.00 condensed in a very small regime at the bottom wall and this may cause bigger heat transfer rates at the bottom wall. It may also be noted that the higher circulation pushes fluid near the upper region of the left wall, and the fluid is pulled away from the central region of the left wall. Consequently, at $R a=10^{4}$, the temperature gradients near the bottom wall are found to be significantly high.

The effects of Prandtl number on average Nusselt number $N u$ at the heated surface and average bulk temperature $\theta_{\text {av }}$ in the Enclosure is illustrated in Fig. $6 \& 7$ with $R e=50$. From this figure, it is found that the average Nusselt number $\mathrm{Nu}$ moves up calmly with increasing $P r$. Moreover, it is also to be highlighted that the highest heat transfer rate occurs for the highest values of $\operatorname{Pr}(=6.0)$. On the other hand, the average bulk temperature of the fluid in the Enclosure increases very sharply in forced dominated region inside the Enclosure and reached to the highest bulk temperature rate at $P r=0.71$. But in the free dominated region bulk temperature rate is slower for the higher values of $P r$.

Table 3: Average Nusselt numbers for different Prandtl number while $\operatorname{Pr}=0.71,2.0,3.0$ and 6.0, $R e=50$.

\begin{tabular}{|c|c|c|c|}
\hline \multicolumn{4}{|c|}{$N u_{\mathrm{av}}$} \\
\hline$P r$ & $R a=10^{3}$ & $R a=5 \times 10^{3}$ & $R a=10^{4}$ \\
\hline 0.71 & 2.248317 & 2.674681 & 3.055404 \\
\hline 2.0 & 3.28083 & 4.045073 & 4.731271 \\
\hline 3.0 & 3.887278 & 4.791325 & 5.577197 \\
\hline 6.0 & 5.162974 & 6.307248 & 7.443361 \\
\hline
\end{tabular}

Table 4: Average bulk temperature for different Prandtl number while $\operatorname{Pr}=0.71,2.0,3.0$ and 6.0, $R e=50$.

\begin{tabular}{|c|c|c|c|}
\hline \multicolumn{4}{|c|}{$\theta_{a v}$} \\
\hline$P r$ & $R a=10^{3}$ & $R a=5 \times 10^{3}$ & $R a=10^{4}$ \\
\hline 0.71 & 0.467239 & 0.585775 & 0.603274 \\
\hline 2.0 & 0.512497 & 0.592877 & 0.590195 \\
\hline 3.0 & 0.518236 & 0.584532 & 0.570561 \\
\hline 6.0 & 0.522066 & 0.558371 & 0.543347 \\
\hline
\end{tabular}



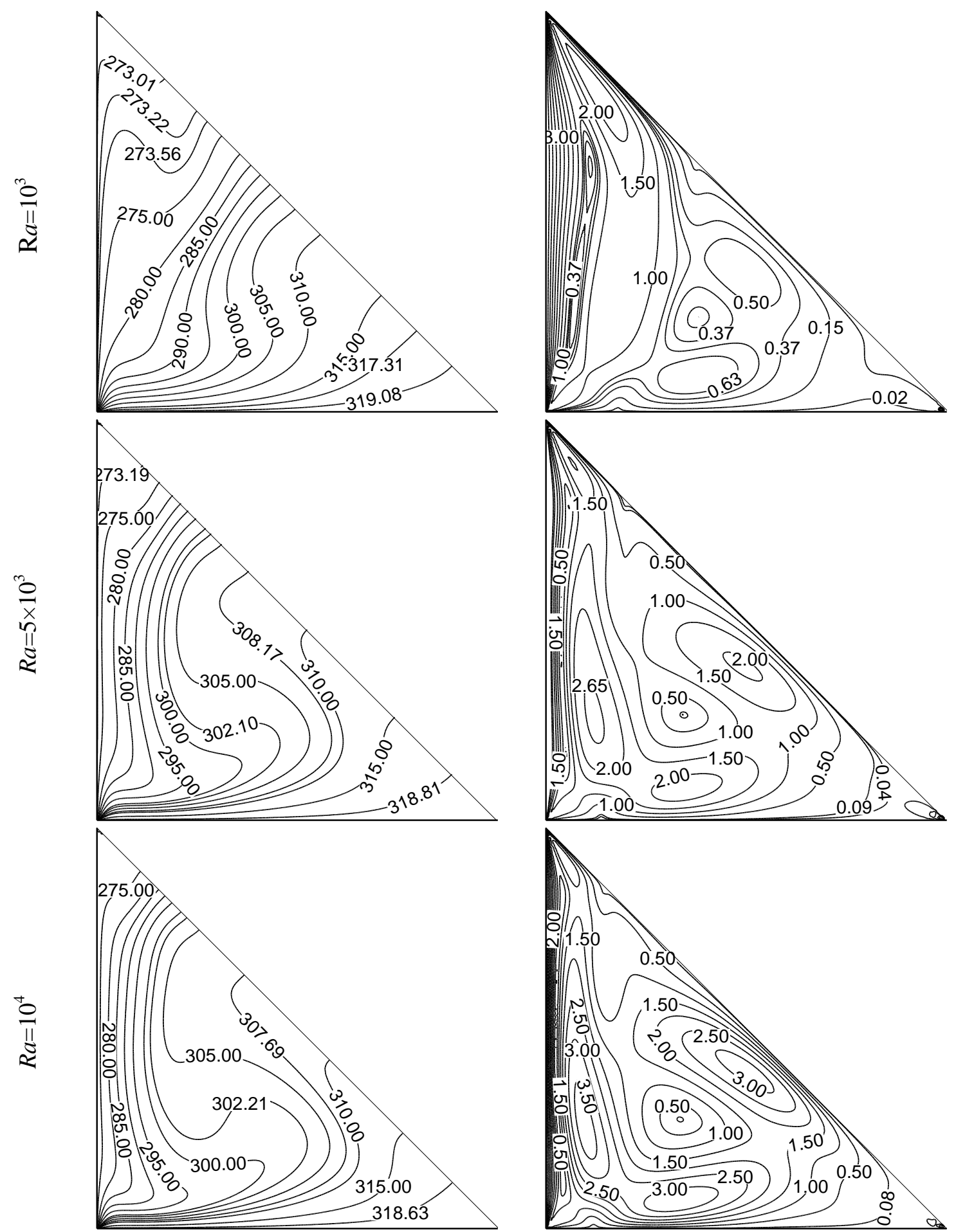

Isotherm

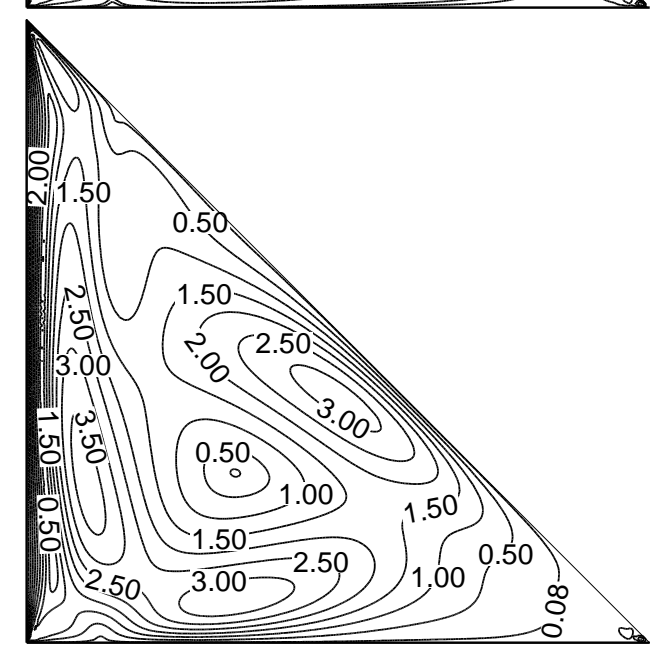

Streamline

Fig 2: Isotherms and streamlines patterns for $R e=50$ and $\operatorname{Pr}=0.71$ 

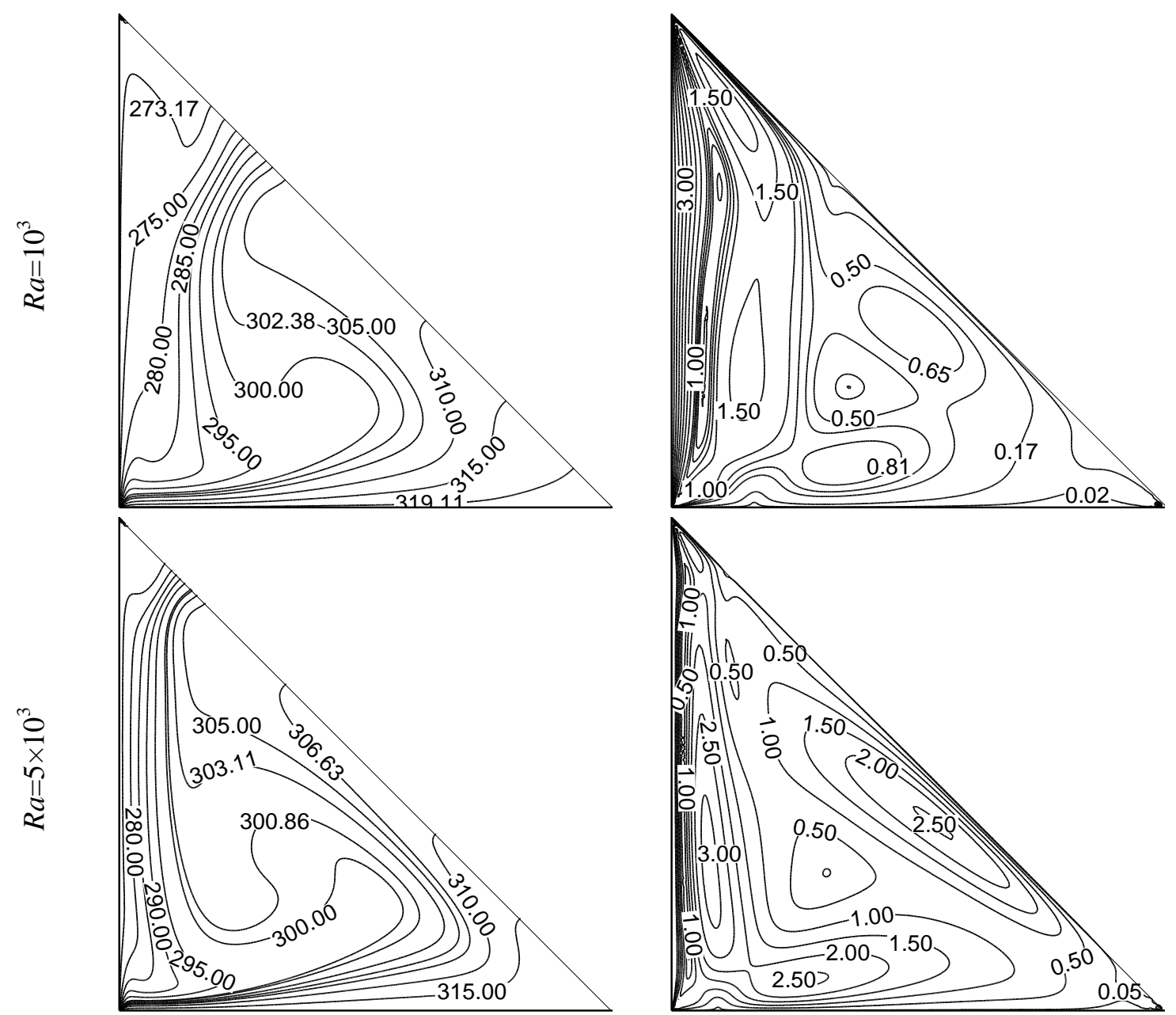

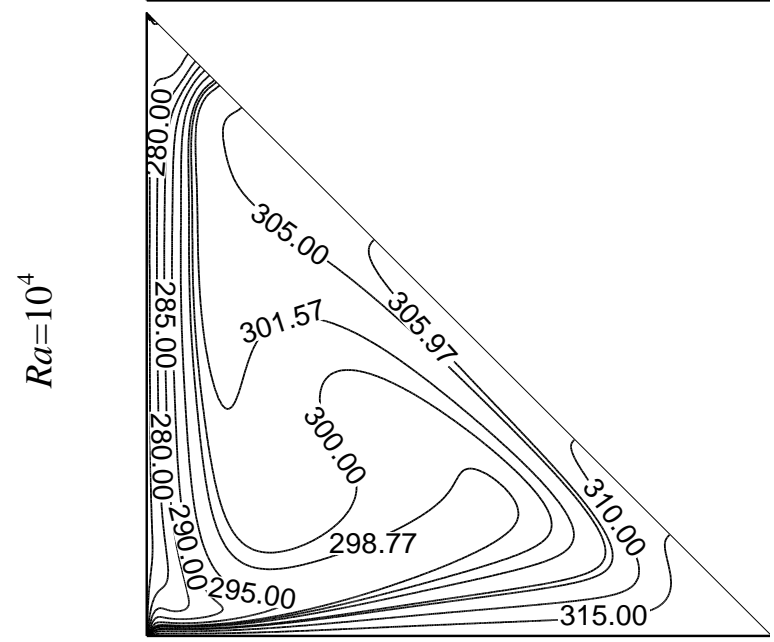

Isotherm

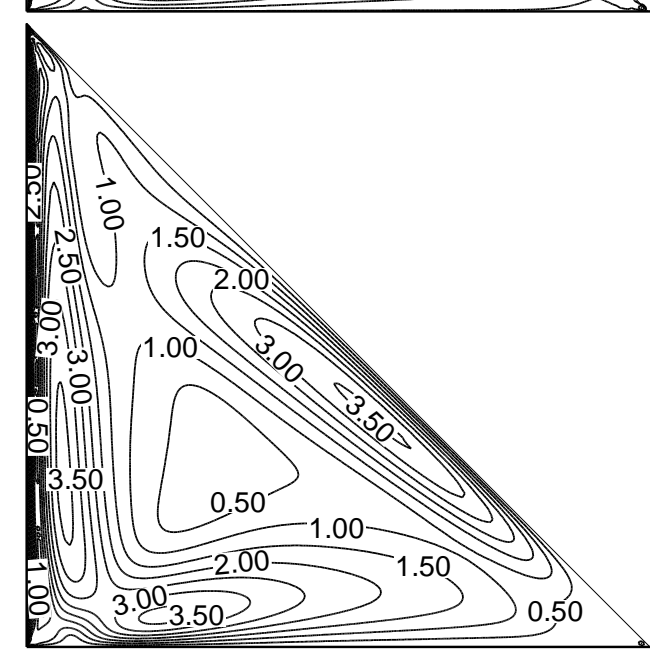

Streamline

Fig 3: Isotherms and streamlines patterns for $\operatorname{Re}=50$ and $\operatorname{Pr}=2$ 

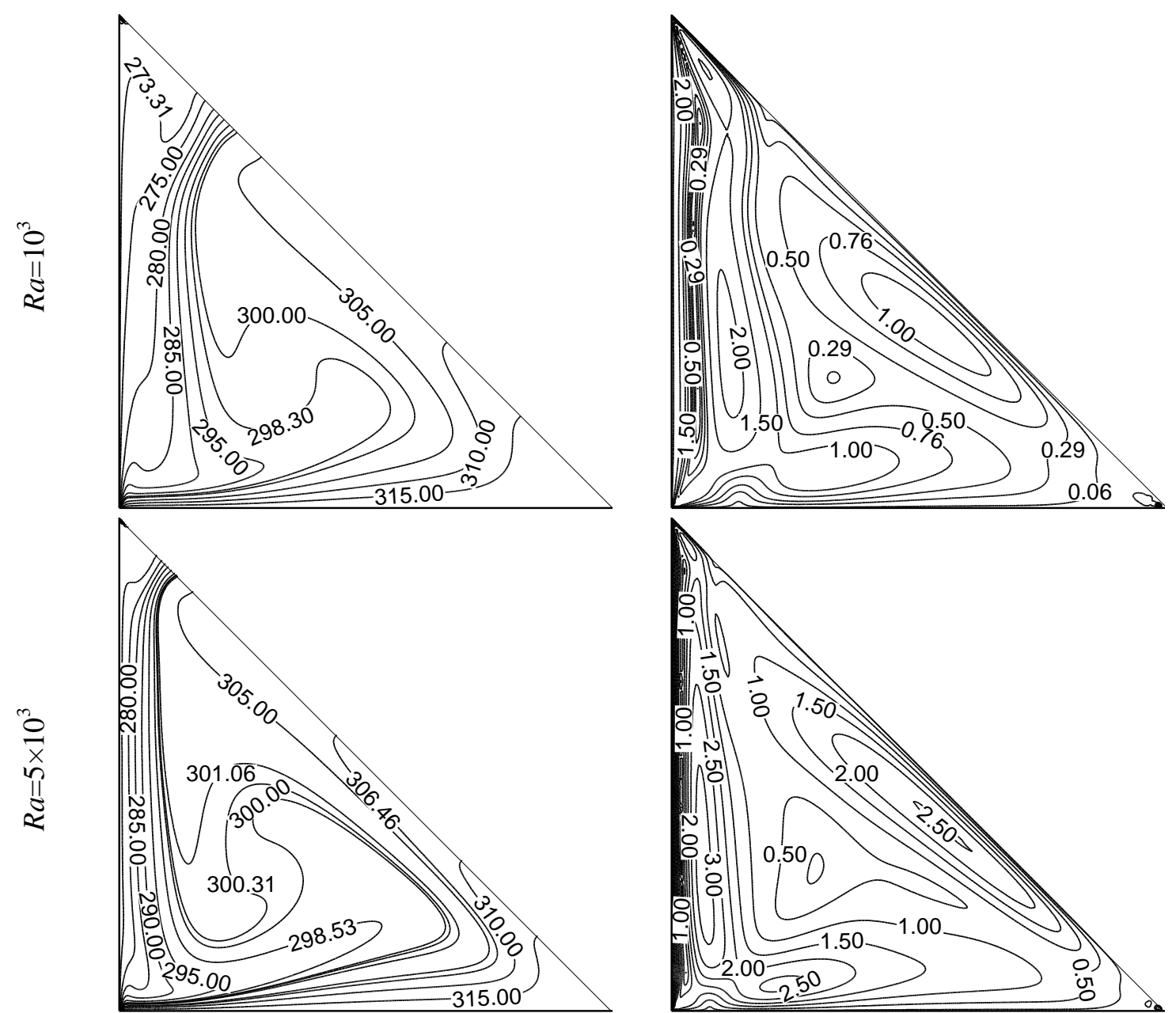

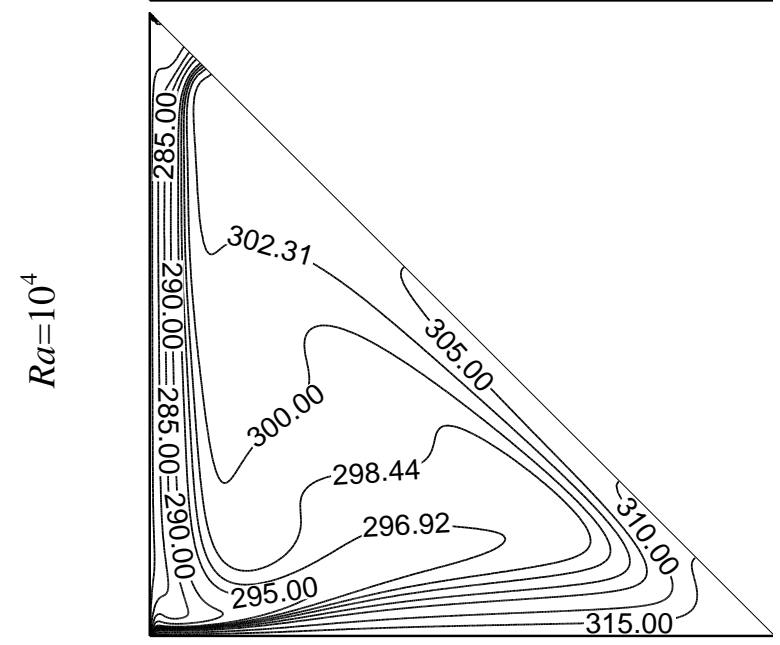

Isotherm

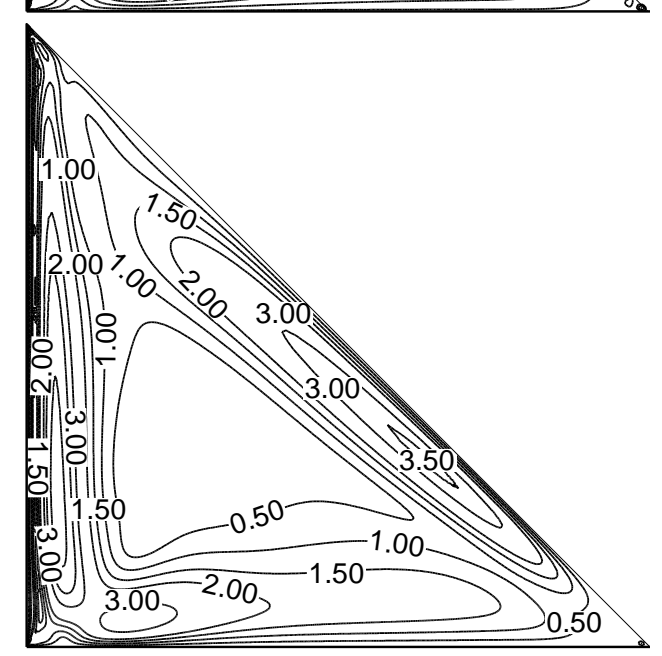

Streamline

Fig 4: Isotherms and streamlines patterns for $\operatorname{Re}=50$ and $\operatorname{Pr}=3$ 

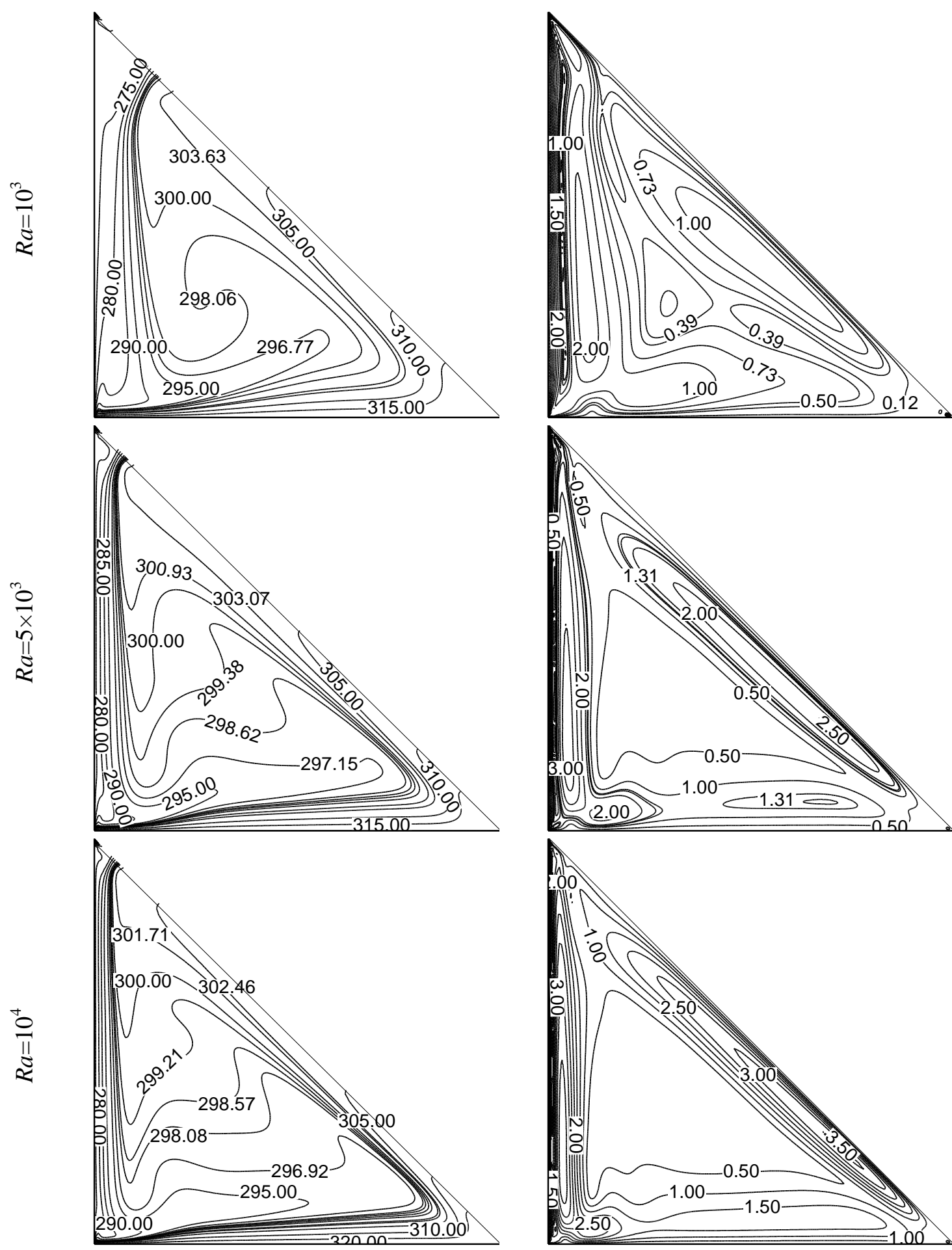

Isotherm

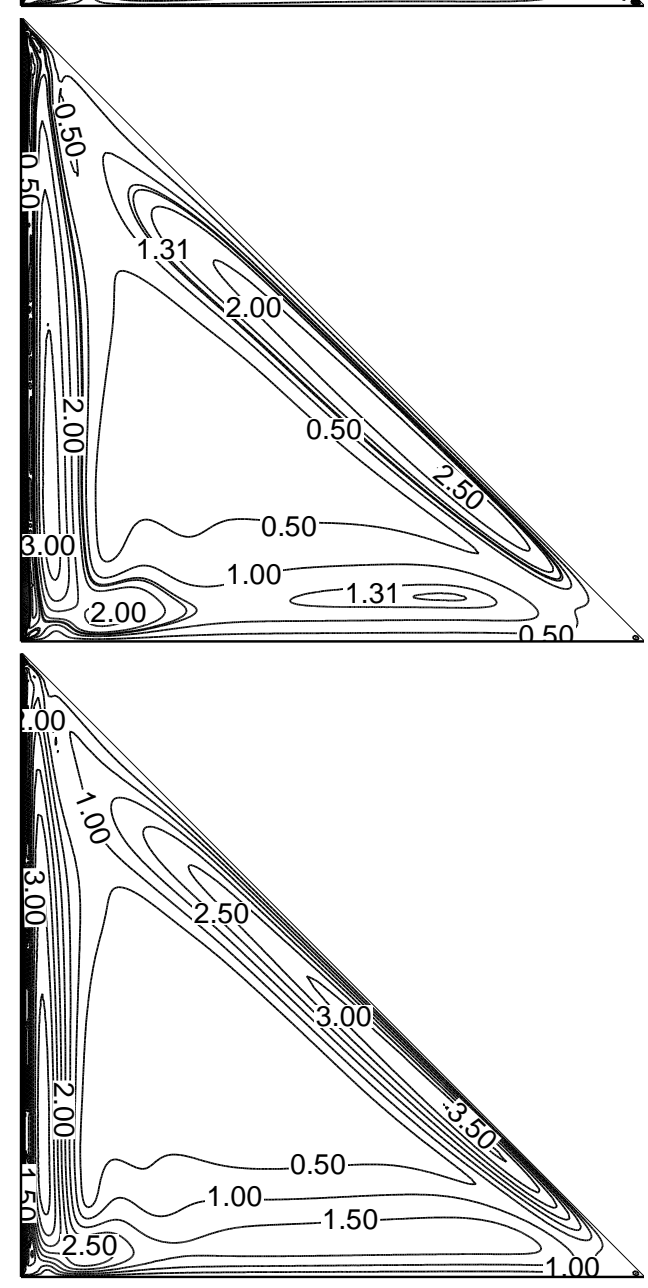

Streamline

Fig 5: Isotherms and streamlines patterns for $R e=50$ and $\operatorname{Pr}=6.0$ 


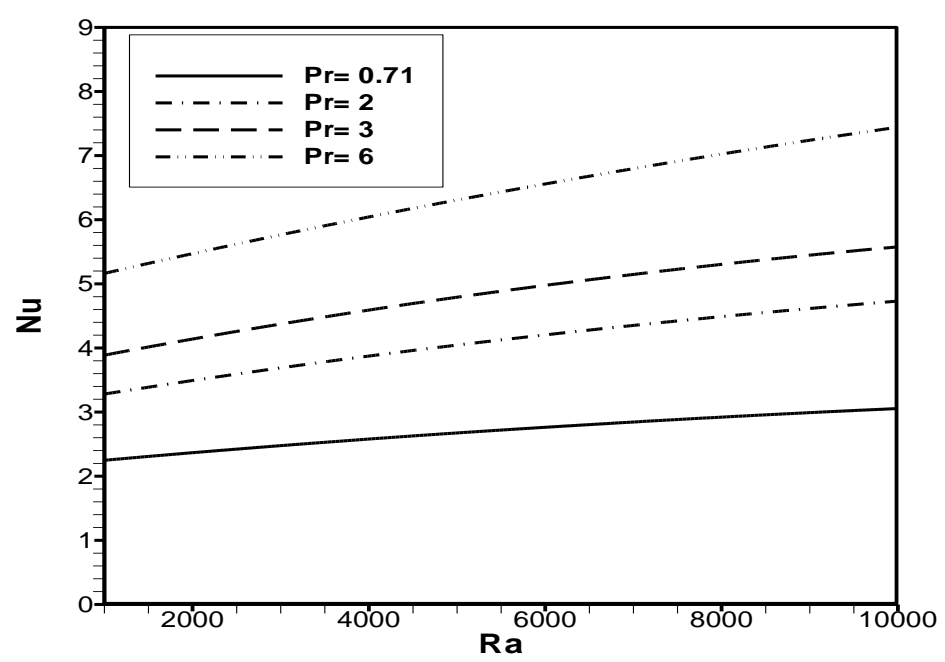

Fig. 6: Effect of average Nusselt number and Rayleigh number while $R e=50$.

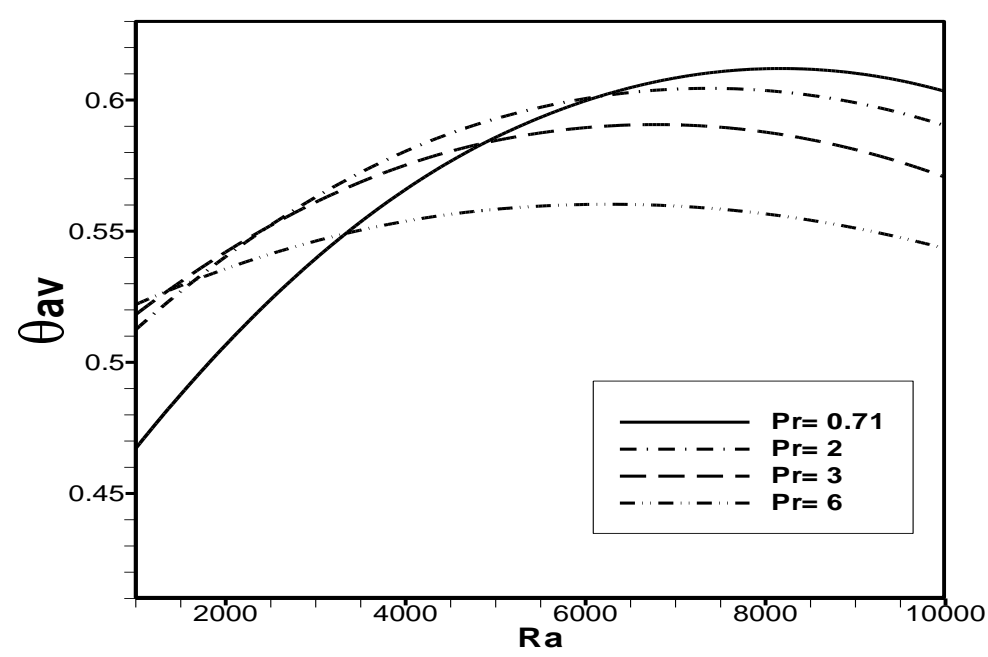

Fig. 7: Effect of average bulk temperature and Rayleigh number while $R e=50$.

\section{Conclusions}

A two-dimensional steady mixed convection flow in a triangular enclosure and subjected to cool at the right vertical wall which is moving, adiabatic conditions at the other inclined wall and the bottom wall are considered hot. In this cases the stream line of flow and thermal fields as well as characteristics of heat transfer process particularly its expansion for the effect of Prandtl number has been evaluated while Reynolds number is fixed varying Rayleigh numbers. On the basis of the analysis the following conclusions have been drawn:

- The impact of Prandtl number on the flow and thermal fields in the Enclosure is found to be more pronounced, if $R e$ is kept fixed. The maximum heat transfer is experimented at the highest value of $\operatorname{Pr}$ for the considered value of $R a$.

- The average bulk temperature is high for $R e=0.71$ in free convection region.

- As $R a$ escalates the value of the magnitude of the stream function is $\psi$ and the cells become larger in size, become more attested to the boundary wall.

- Moreover, the average bulk temperature becomes greatest for $R e=40$ and $R a=5 \times 10^{3}$ and lowest for $R a=10^{4}$. Bulk temperature demonstrations counter behavior with average nusselt number for Reynolds number.

- Thermal and velocity boundary layer thickness is thinner for increasing of Rayleigh number. 
- Several vortex cells are found into the flow field and a secondary vortex at the center and bottom wall of the Enclosure is seen in the streamlines.

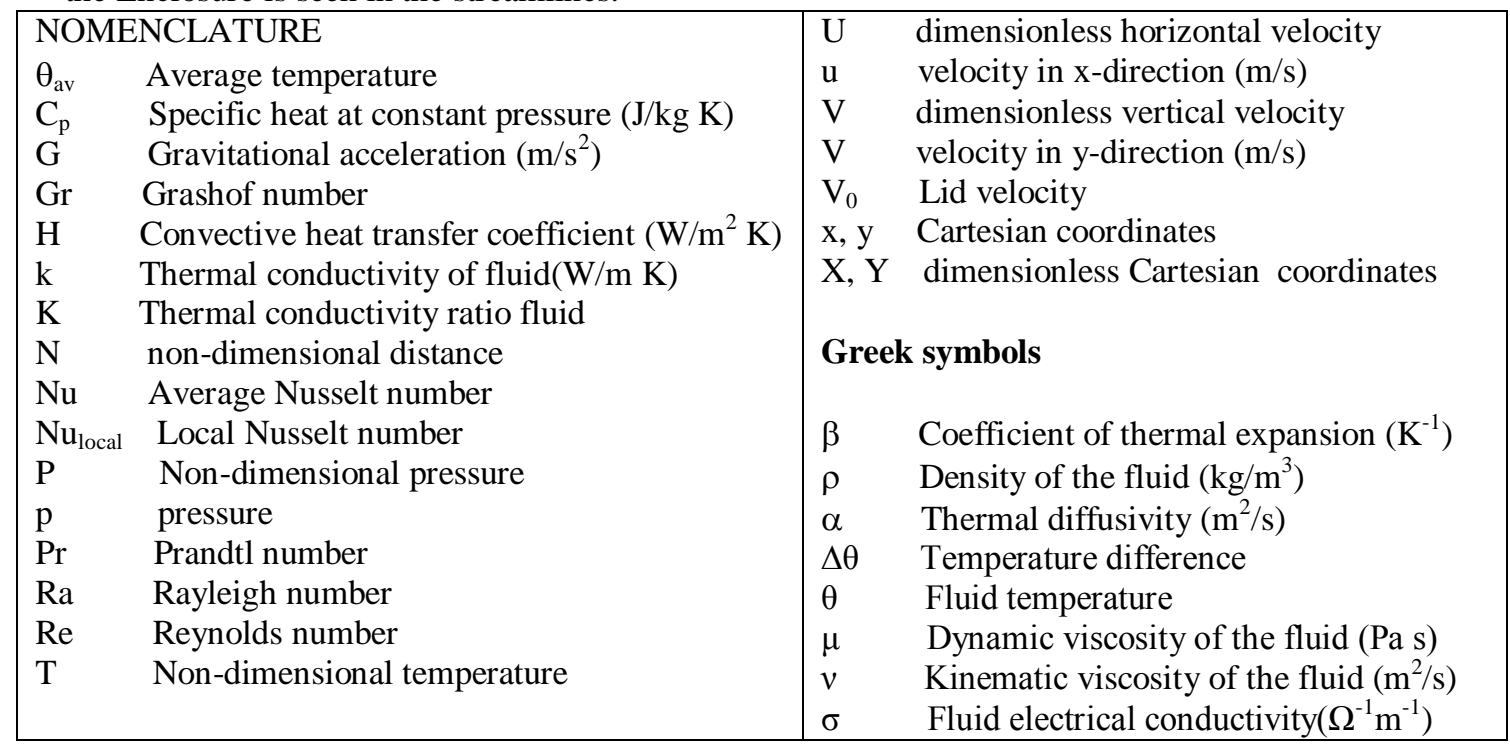

Acknowledgements

Authors would like to express their gratitude to the Department of Arts and Sciences, Ahsanullah University of Science and Technology (AUST), Dhaka, Bangladesh, for providing computing facility during this work.

\section{References}

[1]. G. Biswas, H. Laschefski, N. K. Mitra, and M. Fiebig, "Numerical investigation of mixed convection heat transfer in a horizontal channel with a built in square cylinder", Numer. Heat Transfer, 18, 1990, 173-188.

[2]. A. Raji and M. Hasnaoui, "Mixed convection heat transfer in a rectangular Enclosure ventilated and heated from the side", Numer. Heat Transfer, Part A, 33, 1998a, 533-548.

[3]. A. Raji, and M. Hasnaoui, "Correlations on mixed convection in ventilated Enclosures". Revue Ge'ne'rale de Thermique, 37, 1998 b, 874-884.

[4]. A. Omri, and S. B. Nasrallah, "Control volume finite element numerical simulation of mixed convection in an air-cooled Enclosure", Numer. Heat Transfer, Part A, 36, 1999, 615-637.

[5]. A. Raji, and M. Hasnaoui, "Mixed convection heat transfer in ventilated Enclosures with opposing and assisting flows", Engg. Comp., Int. J. Computer-Aided Eng. Software, 17(5), 2000, 556-572.

[6]. D. Angirasa, "Mixed convection in a vented enclosure with isothermal vertical surface", Fluid Dyn. Res. 26, 2000, 219-233.

[7]. S. Singh, and M. A. R. Sharif, "Mixed convective cooling of a rectangular Enclosure with inlet and exit openings on differentially heated side walls". Numer. Heat Transfer, Part A, 44, 2003, 233-253.

[8]. M. A. Hossain, and R. S. R. Gorla, "Effect of viscous dissipation on mixed convection flow of water near its density maximum in a rectangular enclosure with isothermal wall", Int. J. of Numer. Methods for Heat and Fluid Flow, 16(1), 2006, $5-17$.

[9]. Y. Varol, H.F. Oztop and A. Varol, "Free convection in porous media filled right-angle triangular enclosures", Int. Commun. Heat Mass Transfer, 33, 2006, 1190-1197.

[10]. T. Basak, S. Roy, S. Krishna Babu, A. R. Balakrishnan, "Finite element analysis of natural convection flow in a isosceles triangular enclosure due to uniform and non-uniform heating at the side walls", Int. J. Heat Mass Transfer, 51, 2007, 44964505.

[11]. M. M. Rahman, M. A. Alim, M. A. H. Mamun, M. K. Chowdhury and A. K. M. S. 1slam "Numerical Study of Opposing Mixed Convection in a Vented Enclosure" ARPN J. of Eng. and Applied Sci., 2, 2007, 25-36.

[12]. M. M. Rahman, M. A. Alim, M. M. A. Sarker, Int. Commun. Heat Mass Trans. 37, $2010,524$. http://dx.doi.org/10.1016/j.icheatmasstransfer.2009.12.012

[13]. J.N. Reddy, "An Introduction to the Finite Element Method, McGraw-Hill, New York, 1985. 